Primož Potočnik, Edvard Govekar,

Neural network, ARX, and extreme learning machine models for the short-term prediction of temperature in

buildings,

Building Simulation (2019).

(Available online 10 April 2019)

(c) 2019. This manuscript version is made available under the CC-BY-NC-ND 4.0 license

http://creativecommons.org/licenses/by-nc-nd/4.0/

Link to published article: https://rdcu.be/bxe4b

DOI: https://doi.org/10.1007/s12273-019-0548-y

\title{
Neural network, ARX, and extreme learning machine models for the short-term prediction of temperature in buildings
}

\author{
Primož Potočnik ${ }^{1}$, Boris Vidrih, Andrej Kitanovski, Edvard Govekar \\ University of Ljubljana, Faculty of Mechanical Engineering, 1000 Ljubljana, Slovenia
}

\begin{abstract}
In this paper, the possibilities of developing machine learning based data-driven models for the short-term prediction of indoor temperature within prediction horizons ranging from 1 hour up to 12 hours are systematically investigated. The study was based on a TRNSYS emulation of a residential building heated by a heat pump, combined with measured weather data for a typical winter season in Ljubljana, Slovenia. Autoregressive models with exogenous inputs (ARX), neural network models (NN), and extreme learning machine models (ELM) are considered. The results confirm the finding that nonlinear models, particularly the NN model trained by regularization, consistently outperform linear models in both fitting and generalization performance, so they are the recommended choice as predictive models. The availability of future weather data considerably improved the predictive performance of all the tested models. Besides data about the future outdoor temperature, also data about future expected solar radiation significantly improve predictions of temperature in buildings. The linear models required embedding dimensions of 24 hours for accurate predictions, whereas the nonlinear models were not very sensitive to the use of past data. Nonlinear models required about three months of training data to reach good predictive performance, whereas the linear models converged to accurate predictions within six weeks. The RMSE prediction errors, averaged over all the data sets and all the prediction horizons, are within the range between $0.155^{\circ} \mathrm{C}$ for the linear ARX model (in the case of no future available weather data), and $0.065^{\circ} \mathrm{C}$ for the neural network model (in the case of available future weather data).
\end{abstract}

\section{Keywords}

Predictive models, neural networks, ARX model, extreme learning machines, residential buildings, indoor temperature.

${ }^{1}$ Corresponding author.

E-mail address: primoz.potocnik@fs.uni-lj.si (P. Potočnik) 


\section{Introduction}

New energy efficiency guidelines in the building sector (Zygierewicz 2016) have emphasized the need to reduce final energy consumption. Such a decrease in energy consumption could be achieved, on the one hand, by increasing the thermal efficiency of the building envelope, and, on the other hand, by applying advanced process control techniques to the building services. In many cases, despite the energy efficiency of the building, we are often faced by decreasing indoor thermal comfort and inefficient energy use. This is often caused by inappropriate optimization methods in building services control techniques. The most common traditional optimization methods are based only on the current outdoor temperature where the relation between the outdoor temperature and the temperature of the heating system is defined by the so-called heating curve.

Considerable improvements in the performance of building service systems can be achieved by using advanced process control techniques, such as model predictive control (MPC) (Lindelöf et al. 2015; Serale et al. 2018), where the optimization system consists of a thermal model of the building, weather forecasts, various constraints, and an optimization algorithm. The benefits of applying MPC to building services, both residential and commercial, have been broadly investigated in a number of studies, some of the latest of which are summarized here. For residential buildings, it has been shown that heating demand can be decreased by up to $25 \%$ in the case of high indoor comfort criteria, and by up to $49 \%$ in the case of low indoor comfort criteria (Smarra et al. 2018). A $26 \%$ reduction in heating demand in residential buildings, by using the adaptive predictive control of thermo-active building systems, was presented in (Schmelas et al. 2017), whereas in (Pang et al. 2018) a 16\% reduction in the cooling demand in case of an applied MPC to a radiant slab cooling system was reported. In commercial buildings, the implementation of MPC in building services has resulted in yearly energy savings of between $31 \%$ and $36 \%$ (Killian and Kozek 2018). Somewhat smaller savings are presented in (Bamdad et al. 2018), where the yearly energy saving was up to $26 \%$. The study considering the cold Finnish climate (Arabzadeh et al. 2018) reported that the application of the predictive control could reduce the heating energy cost up to $12 \%$, and concluded that the application of a data-driven control for the demand prediction brings efficiency to demand response control.

In developing MPC for building installation systems, it is essential to select appropriate models for the evaluation of the operation and thermal response of the systems. A number of authors (Afroza et al., article in press; Deb et al. 2017; Do and Cetin 2018; Ahmad et al. 2018) have reported, in their research, the key findings which are relevant to the selection and design of appropriate models for the management of various building installations systems. A comprehensive review of the challenges of building performance simulation was recently presented by (Hong et al. 2018), highlighting some of the most important technical challenges facing building simulation. The crucial component for the effective operation of MPC is the precision and accuracy of the thermal building models (Prívara et al. 2013). There are three general categories of thermal building models: detailed physical models, simplified physical models, and statistically-based models (Li and Wen 2014).

The detailed physical models or so called white-box models (Crawley et al. 2008) are based on physical knowledge about the building and its thermal balance equations. Such models require good knowledge of the structure and of the physical and material properties of the building and the technical properties of the building services. White-box models are appropriate in the case of off-line or preliminary research into new system optimization. The potential benefits of operating a heat pump in an energy-efficient residential building using a white-box model have been presented in (Romero Rodríguez et al. 2018), where cost savings of up to $25 \%$ were achieved by using optimal strategies.

The simplified physical models or grey-box models combine a prior partial physical model with historical data in order to complete or improve the model. The simplified model that was presented in (Reynders et al. 2014) shows, in general, good agreement with detailed physical models. The use of such a model for short- 
term thermal prediction resulted in the best performance with a root-mean square error (RMSE) of less than $0.5^{\circ} \mathrm{C}$ in the case of a prediction horizon of $h=1 \mathrm{~h}$, and up to $1^{\circ} \mathrm{C}$ in the case of a prediction horizon of $h=3$ h (Ferracuti et al. 2017). A better prediction was presented in (Žáčeková et al. 2014) for a real office building, where RMSE values below $0.4^{\circ} \mathrm{C}$ in the spring time and below $0.3^{\circ} \mathrm{C}$ in winter time were reported. In both cases, the prediction horizon was $h=2 \mathrm{~h}$. Using a combination of the RC-model and the 24 hours of historical data resulted in a prediction error in energy use of less than 5\% (Oldewurtel et al. 2012).

It is difficult to apply white-box and grey-box models for on-line operation, since they require thousands of parameters and long training times (Foucquier et al. 2013; Prívara et al. 2013). Besides this, the real building parameters and building service characteristics are often unknown in existing buildings, so that the use of statistical or so called black-box models that have self-learning capabilities is more appropriate (Pedersen 2007; Prívara et al. 2013). Data-driven statistical models which are based on inverse modelling techniques using multiple linear regression analysis between the measured data of the analyzed system (temperature, energy consumption, building occupancy, heat gains, outdoor parameters, etc.) can be successfully applied (Wang et al. 2018; Amasyali and El-Gohary 2018; Wei et al. 2018). The use of artificial intelligence methods, including autoregressive models with exogenous inputs (ARX) and neural networks (NN), has been shown to result in better predictions of building thermal response over traditional regression techniques (Kalogirou 2001; Li et al. 2009). The prediction of 10 min ahead of indoor temperature by means of an ARX model (RíosMoreno et al. 2007) based on $15 \mathrm{~min}$ of historical data of four exterior variables (outdoor temperature, relative humidity, wind velocity and solar radiation) and one interior variable (indoor temperature) showed the best value of the coefficient of determination $\left(\mathrm{R}^{2}\right)$ between 0.88 and 0.90 . Using climate data older than 20 min did not improve the model's performance. Application of an ARX model with two exterior variables (outdoor temperature and solar radiation) and three interior variables (indoor temperature, indoor heat gains, and the thermal gain of the heating/cooling system) showed better accuracy in the case of short-term than long-term prediction of indoor temperature. In the case of 3 hour prediction, the value of RMSE was up to $0.8^{\circ} \mathrm{C}$ and in case of 15 -min prediction up to $0.3^{\circ} \mathrm{C}$ (Ferracuti et al. 2017). A more efficient ARX model (Yun et al. 2012) can be constructed by including a physical understanding of the building capacity (making use of knowledge about energy use for a 4-hour back time step). The results showed that such a model is well suited for the prediction of thermal load for 1-hour ahead. This model is based on four exterior variables (outdoor temperature, relative humidity, wind velocity and solar radiation) and two interior variables (indoor heat gains, and the thermal gain of the heating/cooling system). Regardless of the type of the building and the thermal load (cooling or heating), the accuracy level is between $2.0 \%$ and $5.4 \%$. The outdoor temperature was found to be the most dominant exterior variable.

Using different NN models to predict the daily building and heat pump energy consumption in a residential building (Biswas et al. 2016) showed a satisfactory prediction with $\mathrm{R}^{2}$ values between 0.87 and 0.91 . The models were based on three exterior variables: outdoor temperature, solar radiation and values of the day of the week. In a case study which attempted to optimize hybrid cooling systems (i.e. a combination of a ground heat pump and a cooling tower), different $\mathrm{NN}$ models were used in order to predict the temperature of the cooling water exiting the ground heat exchanger and the cooling tower (Gang et al. 2014). Several exterior and interior variables (the inlet outlet temperature of the cooling water, the operating time, the temperature of the ground, and the ambient temperature, etc.) were taken into account, and the results showed good agreement with the measured data (with an RMSE up to $0.5^{\circ} \mathrm{C}$ ). Development of $\mathrm{NN}$ models to predict indoor temperature and heating energy consumption for residential buildings without knowing the exterior variables was presented in (Magalhães et al. 2017). These models are based on different sets of interior variables which are common for detailed or simplified physical models (indoor temperature, energy consumption, building occupancy, heat gains and thermal load). The authors concluded that the prediction of either heating energy use or indoor temperature was appropriate with $\mathrm{R}^{2}$ higher than 0.93 . One advantage of such models is that they can be used in climatically various areas. Recently, some new machine learning approaches have been 
investigated (Robinson et al. 2017; Liu et al. 2019; Fan et al. 2017; Guo et al. 2018) such as deep learning, extreme learning machines and support vector machines, and good performance of extreme learning machines was reported.

The predictive accuracy of data-driven predictive models is strongly influenced by the availability of exterior and interior variables, and also by the quality and quantity of the training data (i.e. by the length of the training period). The results of one study (Ding et al. 2018) showed that, by using only exterior variables, the prediction accuracy could be up to $12 \%$ lower than in the case when interior variables, too, are used. Based on the limited availability of reports in the literature regarding appropriate lengths of training data, the suggested training periods are between 6 days and 77 days (Ding et al. 2018; Gang et al. 2014; Huang et al. 2018). The training data which are required to train predictive models (i.e. to calculate the model's coefficients) can be obtained either from in-situ data corresponding to real buildings, or from simulation data (i.e. from white-box or greybox models) such as: TRNSYS (Klein et al. 2013; Villa-Arrieta and Sumper 2018), Energy Plus, and others (Crawley et al. 2008; Arabzadeh et al. 2018). The last method based on simulation data is suitable especially in the development of MPC in a case of new buildings, where the required training data (i.e. the thermal response of the building and building service systems) are not yet available.

\subsection{Research objectives}

Based on the results of the performed literature review, we can conclude that there is no universal model that can be utilized under all conditions. The development of the model is therefore essential and requires caseby-case. The main goal of our research was to systematically investigate the possibility of developing machine learning based data-driven models for the short-term prediction of indoor temperature in buildings. The models are designed to be available for application in the model predictive control of thermal comfort in buildings. For this reason short-term predictive horizons within the range from 1 hour to 12 hours were investigated. The specific research topics explored in this paper include:

1. Construction and critical comparison of various data-driven machine learning models, including:

- autoregressive models with external inputs, combined with a stepwise regression procedure for the selection of relevant inputs,

- feedforward neural network based models,

- extreme learning machine models.

2. Investigation of the influence and relevance of the availability of future weather data. The models are constructed in three versions, as follows:

- without any available future weather information,

- with available hourly data about future outdoor temperature,

- with available hourly data about future outdoor temperature and solar radiation.

3. Investigation of the required embedding dimensions for past inputs. Embedding dimensions, denoting the length of recent history of inputs, are explored within the range from 0 to 33 hours, for different model types, and for different availabilities of future weather data.

4. Exploration of the required data length for the construction of models. Various data lengths spanning from 1 week to 6 months were investigated with respect to the generalization ability of the constructed models, with the goal of estimating an appropriate time window, as required for model training in real building situations.

The research results based on all of the above proposed research objectives are considered as new contributions to the field of modelling and the predictive control of thermal comfort in buildings. The first research topic brings new insights into the characteristics of good short-term predictive model structures with respect to their 
complexity and to linearity vs. nonlinearity, and answers the question about the proper choice of the machine learning algorithm.

The second research topic reveals the impact of the availability of future weather data which is important to determine the theoretical boundaries of the predictive accuracy of the developed short-term predictive models with respect to the availability of weather data. In a realistic situation, only weather forecasts are available (and not accurate future weather data), so that the presented results, either including the full availability of future weather data, or without any availability of future weather data, represent the boundaries within which realistic short-term predictions of temperature in buildings can be expected. Furthermore, solar radiation is known to be a significant influential factor for the thermal balance of a building (Soldo et al. 2014), so that this factor was also investigated in the study in order to estimate the potential influence and benefits of the availability of accurate solar radiation forecasts.

The third research topic investigates the required embedding dimensions for all combinations of the models and the availability of weather data. Proper selection of inputs and their embedding dimensions is an important factor in designing predictive models, so that the results with respect to proper embedding dimensions reveal important insights into the causal relationship of building thermal balance with respect to past inputs, and offer practical information for researchers and designers of predictive systems for buildings.

Finally, the required data lengths for the construction of predictive models are analysed, which also helps designers of predictive systems in the planning, constructing and deploying of predictive models for the application of predictive control in buildings. The results of this study systematically reveal the best approach to the construction of short-term predictive models, and are therefore directly applicable to the practical use of predictive models in real buildings.

\section{Simulation data}

In this section, the construction of the TRNSYS building emulator model is described. The weather data for the region of Ljubljana, Slovenia, are then presented, and finally, the preparation of several data sets for this study is described. The data sets were derived by generating heating temperature profiles and combining them with weather data and the TRNSYS emulation. The data sets represent a basis for the training and validation of the predictive models described in this study.

\subsection{The TRNSYS building models}

In this study a single-family residential building was considered. The building, which has two floors and 180 $\mathrm{m}^{2}$ of total living space, has external dimensions of $8 \times 11 \mathrm{~m}$ and an annual specific heating demand of 67 $\mathrm{kWh} / \mathrm{m}^{2}$ year. The heating system consists of a heat pump (air-water) and a floor heating system. The floor heating system is regulated by the inlet temperature of the heating media, i.e. the heating temperature $T_{\text {heat }}$, and the heating is turned off when the heating temperature $T_{\text {heat }}$ drops below the building's indoor temperature $T_{\text {in. }}$. The weather regulated heating curve of the heating system was optimized with the objective of maintaining continuous operation and thereby operating with the highest COP (coefficient of performance) of the heat pump, consequently minimizing the requirements to turn off the heating system. The structures of the external and internal opaque walls were assumed to be of heavyweight construction ("ISO 13790: 2008 Energy performance of buildings - Calculation of energy use for space heating and cooling" 2008). The thermal transmittance of the building envelope was $0.328 \mathrm{~W} / \mathrm{m}^{2} \mathrm{~K}$ for the external wall, $0.167 \mathrm{~W} / \mathrm{m}^{2} \mathrm{~K}$ for the floor, $0.126 \mathrm{~W} / \mathrm{m}^{2} \mathrm{~K}$ for the roof, and $1.3 \mathrm{~W} / \mathrm{m}^{2} \mathrm{~K}$ for the windows. The total solar radiation transmittance of the glazing $(g)$ was 0.551 . The window-to-wall ratio was 0.28 , and the total area of the windows was $58 \mathrm{~m}^{2}$. The windows have external shading devices with a shading factor of 0.75 , and are activated when the solar 
radiation acting on the horizontal plane $(S R)$ exceeds $300 \mathrm{~W} / \mathrm{m}^{2}$ and when the indoor temperature $T_{\text {in }}$ is higher than $24^{\circ} \mathrm{C}$.

The building was considered to be occupied, except during weekdays from 7 AM till 4 PM. During the occupied hours the air exchange rate was $0.5 \mathrm{~h}^{-1}$, whereas during the unoccupied hours the air exchange rate was reduced to $0.2 \mathrm{~h}^{-1}$, which corresponds to the airtightness of the building. The internal heat gains $Q_{\text {in }}$ (people, home appliances and lighting) were estimated to amount to $5 \mathrm{~W} / \mathrm{m}^{2}$ of the building floor area, and were taken into account during the occupied hours. Internal thermal comfort was evaluated by using the predicted mean vote (PMV) model (Fanger 1970). This model takes into account 6 parameters: air temperature, radiant temperature, air speed, humidity, metabolic rate and clothing insulation. The values of the air temperature, radiant temperature and humidity were calculated at each time step, whereas the other parameters of the PMV model were kept constant.

The data sets which are required for the training and testing of the predictive models were calculated using the TRNSYS simulation code (Klein et al. 2013), which is one of the most powerful house energy simulators (Pedersen 2007). The universality, widespread application and validation of TRNSYS has been demonstrated in many studies (Al-Saadi and Zhai 2015; Crawley et al. 2008; Lu et al. 2017; Safa et al. 2015; Drissi et al. 2018) where this model was used to simulate the thermal response of different building structures (e.g. walls, windows, slabs on grade, roofs, and solar walls) in combination with various building services (HVAC, heat pumps, solar systems, etc.). In our study, the main components of the TRNSYS model were: Type 9 (the data read model), Type 16 (the solar radiation processor model), Type 69 (the long-wave radiation exchanger model), Type 33 (the psychometrics model), Type 56b (the multi-zone building model), Type 25 (the printer module), and Type 581 (the multi-dimensional data interpolation model for the heat pump). The accuracy of the TRNSYS models in our study was validated in our previous research (Potočnik et al. 2018), where the adequate performance of TRNSYS emulation was confirmed. We therefore consider the results of the TRNSYS emulation to be accurate and relevant to support the conclusions and recommendations of this study.

\subsection{Weather data}

Measured weather data corresponding to one typical winter season in Ljubljana, Slovenia, were used in this study (ARSO 2017). These data include hourly measurements of the outdoor temperature $T_{\text {out }}$ and the solar radiation $S R$ for a period of six months. Figure 1 shows six months of the measured outdoor temperature $T_{\text {out }}$ and the solar radiation data $S R$.

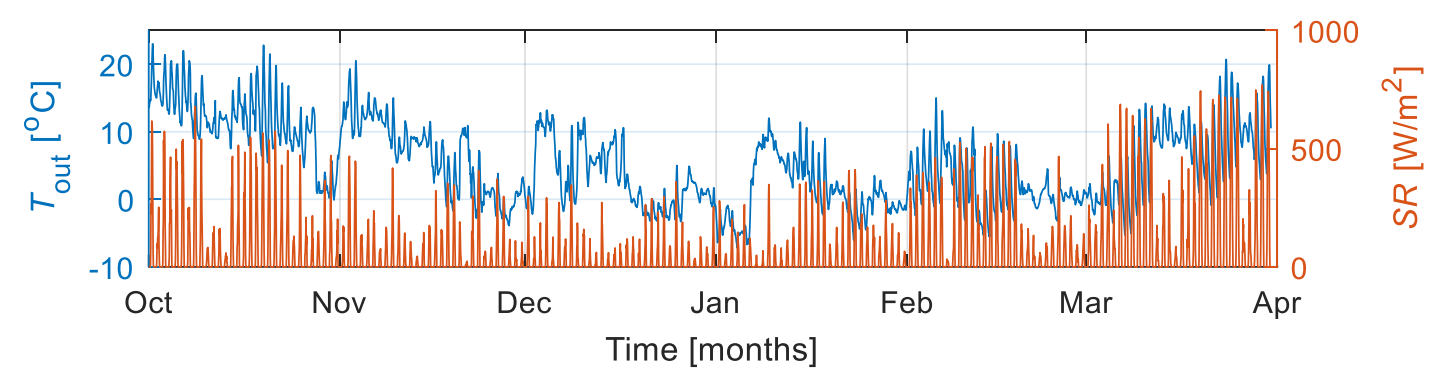

Figure 1: Weather data recorded at Ljubljana, Slovenia, showing the outdoor temperature $T_{\text {out }}$ and the solar radiation $S R$ for a winter period of six months

\subsection{Generating data sets}

This study required several data sets for the training and testing of the investigated predictive models. Each data set consisted of weather data, different heating profiles (control variable $T_{\text {heat }}$ ), and building indoor temperatures $\left(T_{\text {in }}\right)$ calculated by TRNSYS simulations. Different heating temperatures $T_{\text {heat }}$ which corresponded to possible control strategies were generated for each data set. Data sets 1-3 were generated by random $T_{\text {heat }}$ profiles, and data sets 4-6 included $T_{\text {heat }}$ profiles which were optimized according to various 
degrees of freedom in order to achieve minimal (but always positive) PMV values (Potočnik et al. 2018). The data sets are represented as follows:

Data sets 1-3: Randomly generated $T_{\text {heat }}$, with amplitudes ranging between $20^{\circ} \mathrm{C}$ and $35^{\circ} \mathrm{C}$, and time intervals between changes ranging randomly between 1 hour and 96 hours.

Data set 4: Constant $T_{\text {heat }}$ throughout the season, optimized in order to only yield on average the optimal building temperature.

Data set 5: Variable $T_{\text {heat }}$, optimized with respect to the outdoor temperature $T_{\text {out }}$.

Data set 6: Variable $T_{\text {heat }}$, optimized with respect to the outdoor temperature $T_{\text {out }}$ and the solar radiation $S R$.

The data sets are presented in Fig. 2, where the control variable ( $\left.T_{\text {heat }}\right)$ and the building response $\left(T_{\text {in }}\right)$ are shown for each case. The aim of using the proposed data sets was to include a wide range of possible heating regimes, as represented by the randomly generated first three data sets, but also to include more realistic (and actually less demanding) situations that can be encountered in real buildings where measurements can be obtained in already regulated and less flexible heating regimes. The latter is represented by the last three data sets. As described later in Section 4.4, only the first data set was used as a training set to construct the predictive models, and all the other data sets were used as testing data in order to validate the performance of the model.
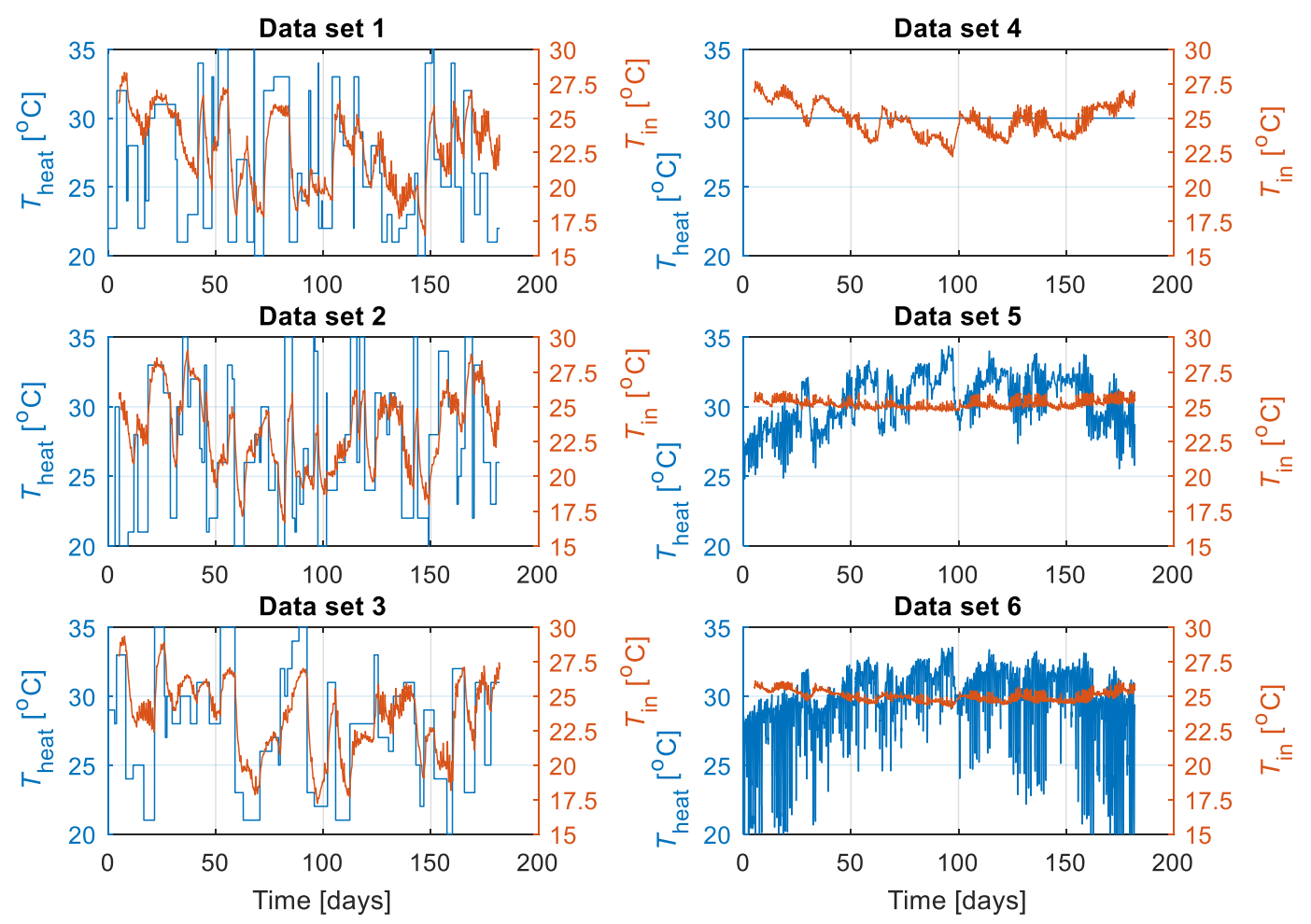

Figure 2: The data sets applied in this study: three data sets $(1,2,3)$ with randomly generated heating temperatures $T_{\text {heat }}$, and three data sets $(4,5,6)$ with increasingly optimized heating temperatures $T_{\text {heat }}$

\subsection{Additional inputs}

Besides the weather-related inputs $\left(T_{\text {out }}, S R\right)$, the internal heat gains $\left(Q_{\text {in }}\right)$, the heating temperatures $\left(T_{\text {heat }}\right)$ and the corresponding building indoor temperatures $\left(T_{\text {in }}\right)$, two further inputs were added in order to facilitate the model's predictive performance. The first additional input was defined as the hour of the day $\left(h_{\mathrm{d}}\right)$, and the second additional input $\left(w_{\mathrm{d}}\right)$ was defined in order to denote a workday or a weekend. The values of these inputs can be $h_{\mathrm{d}}=\{0,1,2, \ldots, 23\}$, and $w_{\mathrm{d}}=\{0,1\}$. By adding these two additional inputs to the created data sets, each complete data set had the structure presented in Table 1. The data sets organized in this format were 
used in our simulation study to construct the predictive models. Table 1 only shows a small subset of data in order to illustrate the data structure. In this study the total number of samples was $N=4369$ for each data set.

Table 1: Example of the data set structure

\begin{tabular}{lllllll}
\hline$h_{\mathrm{d}}[/]$ & $w_{\mathrm{d}}[/]$ & $T_{\text {out }}\left[{ }^{\circ} \mathrm{C}\right]$ & $S R\left[\mathrm{~W} / \mathrm{m}^{2}\right]$ & $Q_{\text {in }}\left[\mathrm{W} / \mathrm{m}^{2}\right]$ & $T_{\text {heat }}\left[{ }^{\circ} \mathrm{C}\right]$ & $T_{\text {in }}\left[{ }^{\circ} \mathrm{C}\right]$ \\
\hline 15 & 0 & 19.3 & 250 & 0 & 21 & 23.3 \\
16 & 0 & 18.9 & 180 & 5 & 21 & 23.6 \\
17 & 0 & 18.0 & 81 & 5 & 21 & 23.7 \\
18 & 0 & 17.0 & 2 & 5 & 21 & 23.4 \\
19 & 0 & 16.2 & 0 & 5 & 21 & 23.4 \\
20 & 0 & 15.3 & 0 & 5 & 21 & 23.3 \\
21 & 0 & 13.9 & 0 & 5 & 26 & 23.2 \\
22 & 0 & 13.7 & 0 & 5 & 26 & 23.1 \\
23 & 0 & 13.0 & 0 & 5 & 30 & 23.1 \\
0 & 1 & 11.5 & 0 & 5 & 27 & 22.9 \\
1 & 1 & 10.3 & 0 & 5 & 27 & 22.8 \\
2 & 1 & 9.4 & 0 & 5 & 20 & 22.7 \\
3 & 1 & 8.6 & 0 & 5 & 22 & 22.6 \\
\hline
\end{tabular}

\section{Methods}

This section provides a brief description of the various data-driven models applied in this study. The model structures included autoregressive models with exogenous inputs (ARX), neural network (NN) models, and extreme learning machine (ELM) models. All of these methods are described in the following subsections. Although support vector machine (SVM) models are another popular and efficient method for the construction of data-driven models (Cortes and Vapnik 1995), this approach was not applied in this study due to difficulty with tuning two of the SVM model parameters, i.e. the generalization parameter $C$, and the kernel parameter $\gamma$. The optimal values of both parameters are arbitrary and should be determined numerically by a crossvalidation method over a wide range of possible values. This complicates the practical application of SVM, since there not much is known about proper values of these two parameters. A comparative analysis of SVM and ELM methods has already shown the similar generalization abilities of both methods (Liu et al. 2012), so by including an ELM model we expected to achieve similar results.

\subsection{ARX model}

The auto-regressive model with exogenous inputs (ARX) is defined by the equation:

$$
A(q) y(t)=B(q) x(t)+e(t)
$$

where $y$ denotes the model output, $x$ the model inputs, $e$ the white noise disturbance, and $A(q)$ and $B(q)$ are polynomials with respect to the time-shift operator $q$ and the model order $M: A(q)=1+a_{1} q^{-1}+\ldots+a_{\mathrm{M}} q^{-\mathrm{M}}$, and $B(q)=\mathrm{b}_{1}+\mathrm{b}_{2} q^{-1}+\ldots+\mathrm{b}_{\mathrm{M}} q^{-\mathrm{M}+1}$.

The linear ARX model can be used to construct a predictive model for the thermal response of a building. In our study, the model order was denoted also by an embedding dimension $D_{\mathrm{e}}$, which denotes the time lag in hours between the moment of prediction and the oldest past inputs. The embedding dimensions of up to $D_{\mathrm{e}}=$ 33 hours used in this study resulted in hundreds of potential inputs, which can result in high model complexity and collinearity problems.

For the above-mentioned reasons, a combination of an ARX structure with a stepwise selection method (Draper and Smith 1998) was used in this study for the optimization of the selection of relevant inputs. In the first step the corresponding ARX model structure with a model order $M=D_{\mathrm{e}}$ was defined, whereas, in the second step, the set of available inputs was reduced by a stepwise regression procedure into only those inputs which are statistically relevant. This results in a compact and robust model, which has good generalization properties. 
Within the context of ARX models the adding of quadratic terms (for each input) was also investigated in order to explore the possible contributions of the nonlinear terms. For this reason two versions of the ARX model were investigated, which in the following text are referred to as:

ARXsr1 - A linear ARX model optimized by stepwise regression

ARXsr2 - A quadratic ARX model optimized by stepwise regression

As an additional option, an ARX model with cross products of all the inputs was also investigated. However, due to the very high number of inputs (and their delayed values) the computations became excessively long (they lasted for more than one day on a computer with an Intel i7-7700 processor and 16 GB of RAM), so that this approach was not considered as a practical solution for real buildings and larger databases.

\subsection{Neural networks}

Within the scope of the research several neural network (NN) models (Haykin 2009) were examined in order to estimate the possible benefits of intrinsically nonlinear model structures compared to ARX models. The different architectures of the NN models were defined as feed-forward networks containing one hidden layer with $N_{\mathrm{h}}$ sigmoid neurons, and an output layer with a linear activation function. The effect of selecting hidden layers of different sizes was explored, and the results are reported below.

The predictor $y$ of a neural network can be represented by a generic expression of the inputs $x$, e.g. for a network with a $K$-dimensional input $x=\left\{x_{1}, x_{2}, \ldots, x_{K}\right\}, N_{\mathrm{h}}$ neurons in the single hidden layer, and a single output, the model can be described as:

$$
y=f_{\mathrm{o}}\left(\sum_{j=0}^{N_{h}} w_{j} f_{h}\left(\sum_{i=0}^{K} w_{j i} x_{i}\right)\right)
$$

where $f_{\mathrm{o}}$ represents the linear output layer activation function, and $f_{\mathrm{h}}$ the sigmoid hidden layer activation function. If past outputs are also included as inputs to the model, then such a structure can be referred to as a nonlinear ARX model, or a "NARX" model.

Two different training methods were used: the Levenberg-Marquardt training algorithm (LM) (Hagan and Menhaj 1994), and the same algorithm with Bayesian regularization (BR) (Foresee and Hagan 1997). Whereas the first method is a standard training method for smaller NN models, the second often results in improved generalization properties due to regularization. For this reason two types of NN models were used, as follows (the numbers denote the size of the hidden layer):

NNIm4 - A neural network with 4 hidden neurons, trained by the LM algorithm

NNbr6 - A neural network with 6 hidden neurons, trained by the BR algorithm

\subsection{Extreme learning machines}

Extreme learning machines (ELM) represent a new fast-learning algorithm for single layer feed-forward networks (Huang et al. 2006). The ELM algorithm randomly chooses hidden layer nodes, and analytically determines the network's output weights. It tends to provide good generalization performance at an extremely fast learning speed. A broad overview of various ELM applications has been summarized in (Ding et al. 2014), and an extensive review of the current state of ELM has been presented in (Huang et al. 2015). An ELM model can be defined by the following expression: 


$$
y=\sum_{j=1}^{N_{h}} w_{j} F_{h}(x)
$$

where $w_{j}$ represents the output weights, and $F_{\mathrm{h}}$ the nonlinear feature mapping, which is defined as a nonlinear piecewise continuous function which satisfies the ELM universal approximation theorems. Commonly used ELM mapping functions include sigmoid, hyperbolic tangent, Gaussian, multiquadratic, and cosine functions. ELM train single-layer feed-forward networks in two main stages:

1) random feature mapping, and

2) solving the linear parameters.

In the first stage, the ELM randomly initializes the hidden layer in order to map the input data into an ELM feature space by means of the nonlinear mapping functions $\left(F_{\mathrm{h}}\right)$. In the second stage of ELM learning, the weights $w_{j}$ which connect the hidden layer and the output layer are solved by minimizing the approximation error in the squared error sense which leads to a global solution.

In comparison with NN models, ELM models generally require a larger number of hidden layer neurons in order to achieve good approximation and generalization properties. In our study, sigmoidal mapping functions were used in the hidden layers, and the models were denoted like this:

ELM300 - Extreme learning machine model with 300 sigmoidal neurons.

\section{Identification procedures}

Based on the generated data sets as described in Section 2, and using the various data-driven models presented in Section 0, a series of numerical experiments were performed in order to explore specific aspects of the prediction of thermal response in buildings. Following the research objectives stated in Subsection 1.1, ARX, $\mathrm{NN}$ and ELM model structures were explored with respect to the effects of the availability of future weather data, embedding dimensions, and the required data lengths for the construction of predicting models.

\subsection{Prediction horizons}

Short-term predictive models were investigated, meaning that the models were constructed for hourly predictions with prediction horizons $h$ within the range from 1 hour to 12 hours: $h=\{1,2, \ldots, 12\}$ hours. A separate model was constructed for each prediction horizon $h$, so that each model structure, such as ARXsr1, refers to a set of 12 models constructed by the same method but for different prediction horizons.

\subsection{Selection of inputs}

Here some comments are given with regard to the composition of inputs and outputs for the predictive models from the data sets. This composition is illustrated by means of the data shown in Table 1, and for the following situation: predicting at time $8 \mathrm{PM}$ for $h=5$ hours ahead, and applying the embedding dimension $D_{\mathrm{e}}=3$. Table 2 illustrates the composition of the inputs and outputs for three future weather conditions. The time stamp at $h_{\mathrm{d}}=20$ represents the moment of prediction - now (corresponding to $8 \mathrm{PM}$ ), and the data available at $h_{\mathrm{d}}=20$ are the last available actual data. Since this example refers to the prediction at $h=5$, the output for all cases is an indoor temperature $\left(T_{\text {in }}\right) 5$ hours ahead from now, i.e $T_{\text {in }}$ at $h_{\mathrm{d}}=1$, with a value of $22.8^{\circ} \mathrm{C}$. The output is marked in the table in yellow.

Applying the embedding dimension $D_{\mathrm{e}}=3$ means that past inputs, up to 3 hours ago, are also used and are marked in blue. The heating temperature $T_{\text {heat }}$ is the control variable, and is therefore also available in the future until one step less than the prediction horizon (this would be a typical situation in a model predictive 
control application). The two additional inputs $h_{\mathrm{d}}$ and $w_{\mathrm{d}}$ are also available in the future because the moment of prediction is known. The past values of these two descriptors are not necessary, so they are not used as inputs. The three future weather condition cases are only differentiated by the availability of future weather related values. Therefore, in comparison with case I, case II additionally includes also future outdoor temperatures $T_{\text {out, }}$ and case III future solar radiation values $S R$. All the future inputs are marked in green.

Table 2: Composition of the inputs and outputs for three future weather conditions, and for a time of prediction $h_{\mathrm{d}}=20$, a horizon $h=5$, and an embedding dimension $D_{\mathrm{e}}=3$.

\begin{tabular}{|rrrrrrr|}
\hline \multicolumn{7}{|c}{ Case I: No future weather data } \\
\hline \hline$h_{\mathrm{d}}$ & $w_{\mathrm{d}}$ & $T_{\text {out }}$ & $S R$ & $Q_{\text {in }}$ & $T_{\text {heat }}$ & $T_{\text {in }}$ \\
\hline 15 & 0 & 19 & 250 & 0 & 21 & 23.3 \\
16 & 0 & 19 & 180 & 5 & 21 & 23.6 \\
17 & 0 & 18 & 81 & 5 & 21 & 23.7 \\
18 & 0 & 17 & 2 & 5 & 21 & 23.4 \\
19 & 0 & 16 & 0 & 5 & 21 & 23.4 \\
20 & 0 & 15 & 0 & 5 & 21 & 23.3 \\
21 & 0 & 14 & 0 & 5 & 26 & 23.2 \\
22 & 0 & 14 & 0 & 5 & 26 & 23.1 \\
23 & 0 & 13 & 0 & 5 & 30 & 23.1 \\
0 & 1 & 12 & 0 & 5 & 27 & 22.9 \\
1 & 1 & 10 & 0 & 5 & 27 & 22.8 \\
2 & 1 & 9.4 & 0 & 5 & 20 & 22.7 \\
3 & 1 & 8.6 & 0 & 5 & 22 & 22.6 \\
\hline
\end{tabular}

\begin{tabular}{|rrrrrrr|}
\hline \multicolumn{7}{|c}{ Case II: Available future $T_{\text {out }}$} \\
\hline \hline 15 & 0 & 19 & 250 & 0 & 21 & 23.3 \\
16 & 0 & 19 & 180 & 5 & 21 & 23.6 \\
17 & 0 & 18 & 81 & 5 & 21 & 23.7 \\
18 & 0 & 17 & 2 & 5 & 21 & 23.4 \\
19 & 0 & 16 & 0 & 5 & 21 & 23.4 \\
20 & 0 & 15 & 0 & 5 & 21 & 23.3 \\
21 & 0 & 14 & 0 & 5 & 26 & 23.2 \\
22 & 0 & 14 & 0 & 5 & 26 & 23.1 \\
23 & 0 & 13 & 0 & 5 & 30 & 23.1 \\
0 & 1 & 12 & 0 & 5 & 27 & 22.9 \\
\hline 1 & 1 & 10 & 0 & 5 & 27 & 22.8 \\
2 & 1 & 9.4 & 0 & 5 & 20 & 22.7 \\
3 & 1 & 8.6 & 0 & 5 & 22 & 22.6 \\
\hline
\end{tabular}

\begin{tabular}{|rrrrrrr|}
\hline \multicolumn{7}{|c}{ Case III: Available future $\boldsymbol{T}_{\text {out }}$ and $\boldsymbol{S} \boldsymbol{R}$} \\
\hline \hline 15 & 0 & 19 & 250 & 0 & 21 & 23.3 \\
16 & 0 & 19 & 180 & 5 & 21 & 23.6 \\
17 & 0 & 18 & 81 & 5 & 21 & 23.7 \\
18 & 0 & 17 & 2 & 5 & 21 & 23.4 \\
19 & 0 & 16 & 0 & 5 & 21 & 23.4 \\
20 & 0 & 15 & 0 & 5 & 21 & 23.3 \\
21 & 0 & 14 & 0 & 5 & 26 & 23.2 \\
22 & 0 & 14 & 0 & 5 & 26 & 23.1 \\
23 & 0 & 13 & 0 & 5 & 30 & 23.1 \\
0 & 1 & 12 & 0 & 5 & 27 & 22.9 \\
\hline 1 & 1 & 10 & 0 & 5 & 27 & 22.8 \\
2 & 1 & 9.4 & 0 & 5 & 20 & 22.7 \\
3 & 1 & 8.6 & 0 & 5 & 22 & 22.6 \\
\hline
\end{tabular}

Legend:

Past inputs

Future inputs

Output

\subsection{Performance measure}

The root mean square error measure was used as the basic performance measure:

$$
e_{\mathrm{RMS}}=\sqrt{\frac{1}{\mathrm{~N}} \sum_{k=1}^{N} e(k)^{2}}
$$

where $N$ denotes the number of samples and $e(k)$ the error between the predicted and the actual indoor temperature (the output of TRNSYS emulation): $e(k)=\widehat{T}_{i n}(k)-T_{i n}(k)$. The root mean square error is a suitable measure for use in building temperature predictions due to its quadratic sensitivity to prediction discrepancies: It is expressed in $\left[{ }^{\circ} \mathrm{C}\right]$ units and thus also can provide a good intuitive evaluation of the model performance. Since human sensitivity to temperature variations is in the order of $\approx 0.1^{\circ} \mathrm{C}$, this level represents a suitable basis for the evaluation of a model's performance, since we are trying to construct models with a prediction accuracy that is close to human temperature sensitivity.

The basic $e_{\mathrm{RMS}}$ measure was calculated for each model, and for every prediction horizon $h$. In order to summarize a model's performance, the basic measures were averaged across all the prediction horizons $h=$ $\{1,2, \ldots, 12\}$ and the averaged RMSE measure, expressed in $\left[{ }^{\circ} \mathrm{C}\right]$, was used as the final performance measure:

$$
\operatorname{RMSE}=\frac{1}{12} \sum_{h=1}^{12} e_{\mathrm{RMS}}(h)
$$

\subsection{Cross-validation}

Since in this study the generalization performance of each model had to be evaluated, an intensive crossvalidation method was applied in which only a single data set was used as a training data (data set 1), and all 
the other data sets $(2,3, \ldots, 6)$ were used as testing data. Using this procedure, it was possible to assess each model's generalization performance across various heating regimes, so that a good estimation of the quality of each model could be obtained.

Based on this strong cross-validation approach, the final model performance was determined by calculating the average performance measure RMSE across all 6 data sets (1 training data set, and 5 testing data sets). This averaged performance measure was applied, and reported in all the subsequent model evaluation results.

\subsection{Identification protocol}

The identification protocol can be summarized as follows:

1. Define the model structure: ARXsr1, ARXsr2, NNlm, NNbr, ELM

2. Define the future weather conditions:

- no available future weather data,

- future values of $T_{\text {out }}$ are available,

- future values of $T_{\text {out }}$ and $S R$ are available

3. Define the embedding dimension for past inputs:

$$
D_{\mathrm{e}}=\{0,3,6, \ldots, 33\}
$$

4. Define the data length for model training (W denoting weeks, and $\mathrm{M}$ months): $1 \mathrm{~W}, 2 \mathrm{~W}, 3 \mathrm{~W}, 4 \mathrm{~W}, 5 \mathrm{~W}, 6 \mathrm{~W}, 2 \mathrm{M}, 3 \mathrm{M}, 4 \mathrm{M}, 5 \mathrm{M}, 6 \mathrm{M}$.

5. For each prediction horizon $h=\{1,2, \ldots, 12\}$ :

- compose the model inputs and outputs (according to the above definitions),

- construct and train the model using the training data (data set 1),

- test the model with regard to all the data (data sets $1,2, \ldots, 6$ ),

- calculate the basic error measure $e_{\mathrm{RMS}}$ (for each prediction horizon, and for each data set).

6. Average the basic error measures $e_{\mathrm{RMS}}$ across all the prediction horizons, and all the data sets, in order to obtain the final averaged RMSE performance measure.

The above-defined identification protocol was adapted in order to be able to include several specific investigation objectives of this paper, where usually only one parameter (such as an embedding dimension or a length of data) was left open, and the other parameters were fixed. In the next section of this paper the results of the performed research are presented.

\section{$5 \quad$ Results and discussion}

\subsection{Prediction horizons}

The first result shown in Fig. 3 illustrates the prediction accuracy over all the prediction horizons $h=\{1,2$, $\ldots, 12\}$ for two model structures (ARXsr1 and NNbr6). This result was obtained by using the second future weather condition case (available data about future outdoor temperatures), an embedding dimension $D_{\mathrm{e}}=24$, and a full data length $(6 \mathrm{M})$. The prediction accuracy is, as expected, the best for the nearest prediction horizon $(h=1)$, and decreases with the increasing distance of the horizon. Compared to the linear ARX model, the neural network model trained by the BR algorithm consistently generates more accurate predictions, and has comparable variance across the data sets. All the subsequent results are shown in compact form, averaged over all the prediction horizons and all the data sets, and are therefore expressed by the averaged performance 
measure (RMSE). For the case shown in Fig. 3, the averaged performance measures amount to RMSE = $0.124^{\circ} \mathrm{C}$ for the ARXsr 1 model, and $\mathrm{RMSE}=0.091^{\circ} \mathrm{C}$ for the NNbr6 model.
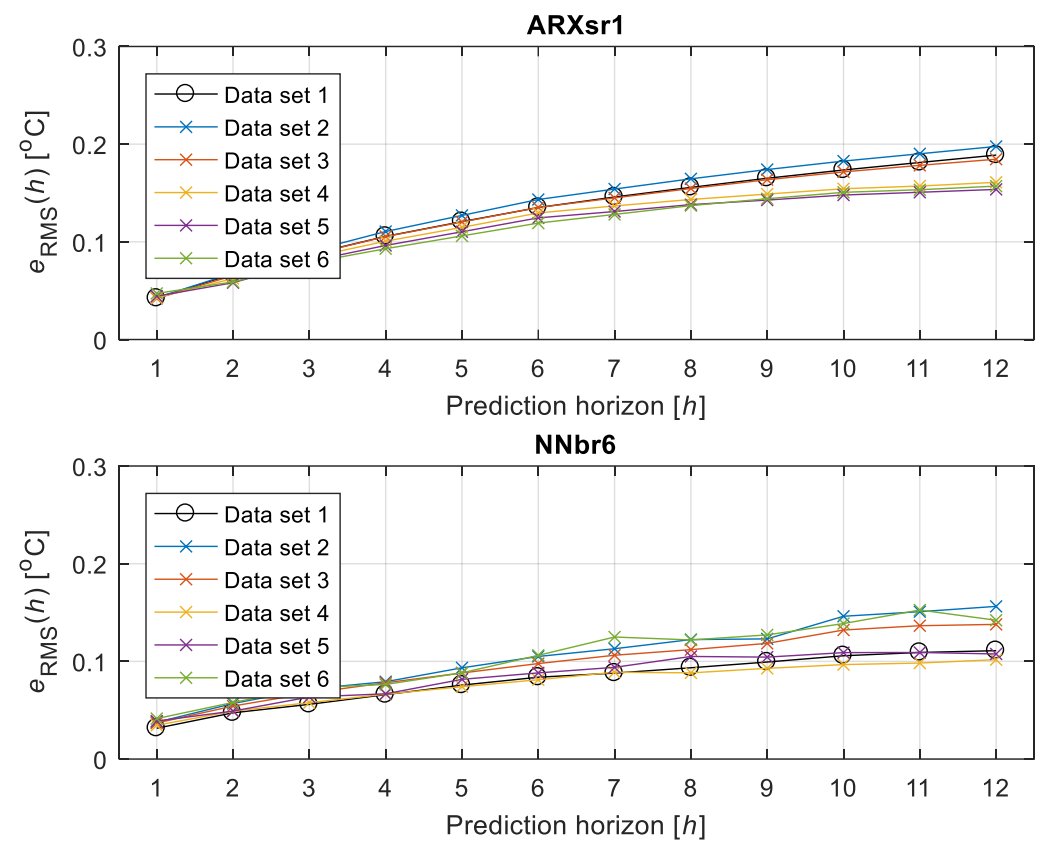

Figure 3: Prediction errors for the weather condition case where there are available data about the future values of $T_{\text {out }}$, for the ARXsr1 and NNbr6 models, and for all the prediction horizons $h=1,2, . ., 12$

\subsection{The topology of the nonlinear models}

The construction of linear ARXsr models is relatively easy and does not require the selection or tuning of special parameters. On the other hand, nonlinear data-driven models usually require the definition of various structural parameters (e.g. about the topology, activation functions, and connections). In our study, NN and ELM models were implemented, both of which require the definition of hidden layer activation functions and the number $N_{\mathrm{h}}$ of hidden layer neurons. Standard sigmoid transfer functions were selected for both of the nonlinear models, and a suitable number $N_{\mathrm{h}}$ of hidden layer neurons was determined experimentally. In Fig. 4, the solutions for three sets of future weather conditions are shown, for the NNbr and ELM models. Due to the very different nature of these two model structures, the results, too, differed considerably. For the neural network models trained by BR, about 5 hidden neurons are enough for good quality predictions. The minima are very flat, which means that the number of hidden neurons is not a critical parameter, so that good NN models can be quite easily constructed by less experienced users. In the case of extreme learning machine models, many more neurons are required due to randomly distributed hidden layer weights. The results stabilize at around 200 units, with only small improvements when $N_{\mathrm{h}}$ is further increased. Based on these results, further experiments were performed with $N_{\mathrm{h}}=6$ for the NNbr models, and $N_{\mathrm{h}}=300$ for the ELM models. The NN trained by the LM algorithm requires even fewer hidden neurons, so that $N_{\mathrm{h}}=4$ was applied for the NNlm models. The corresponding models were denoted as NNbr6, ELM300, and NNlm4. 

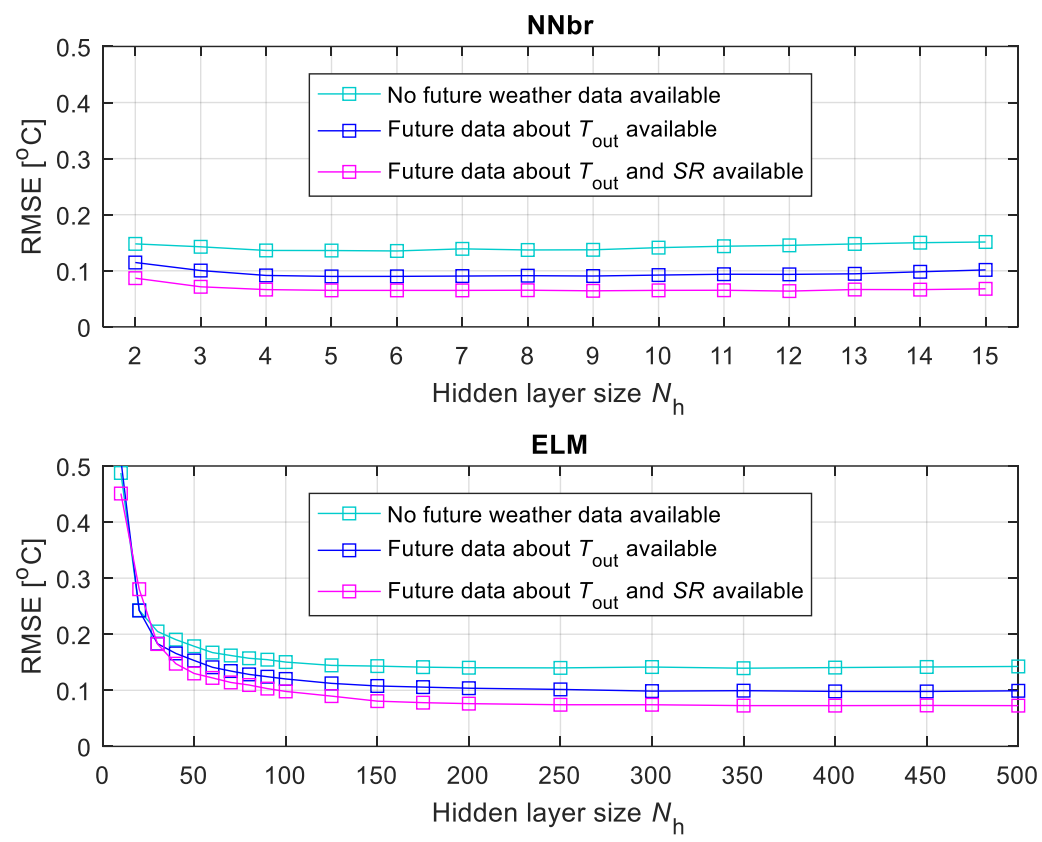

Figure 4: RMSE performance measures for the neural network models (NNbr) and extreme learning machines models (ELM), for a different number of hidden neurons $N_{\mathrm{h}}$

\subsection{Embedding dimension}

The embedding dimension $D_{\mathrm{e}}$ defines the time lag in hours between the moment of prediction and the oldest past inputs. Models with larger embedding dimensions can therefore provide greater expressive power which can be used to capture the dynamics of the predicted phenomena, but this also considerably increases the model's complexity. Consequently, the definition of proper embedding dimensions is an important part of the design of predictive models. For this reason embedding dimensions within the range from 0 to 33 hours, $D_{\mathrm{e}}=$ $\{0,3,6, \ldots, 33\}$, were explored for different model types, and for the different availability of data about future weather conditions. The embedding dimension $D_{\mathrm{e}}=0$ corresponds to the situation where only present data are used, without consideration of any past inputs.

Figure 5 shows the performance measures for two of the investigated models, i.e. the linear ARXsr1 model, and the nonlinear NNbr6 model. The linear model requires considerably larger embedding dimensions for accurate predictions. With respect to the availability of future weather data, the linear models converge to stable predictions at $D_{\mathrm{e}}=24$ hours. The nonlinear NN models are not very sensitive to the utilization of past data, but still benefit if recent data obtained within the last day are included. Consequently, the embedding dimension $D_{\mathrm{e}}=24 \mathrm{~h}$ was used in subsequent experiments when exploring the required data lengths, and comparing all the available predictive models in a uniform manner. 

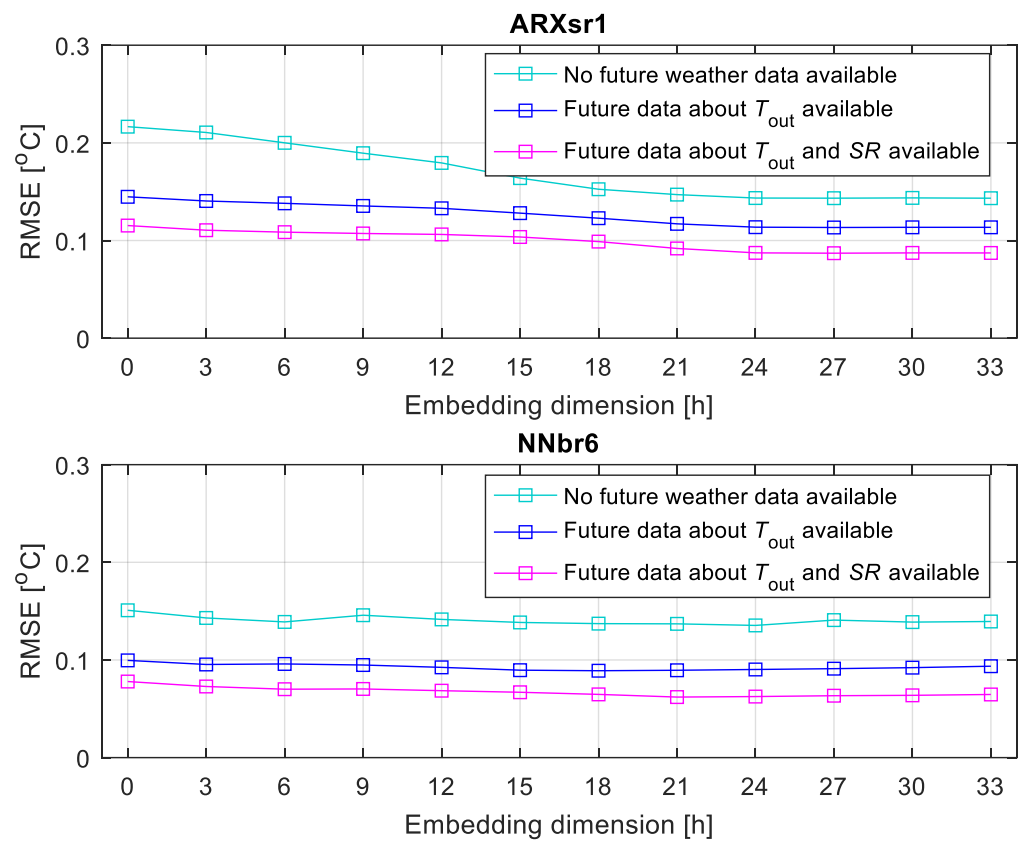

Figure 5: RMSE performance measures for the ARXsr1 and NNbr6 models, for different embedding dimensions $D_{\mathrm{e}}=$ $\{0,3, \ldots, 33\}$ hours

\subsection{Data length}

The expression "required data length" means how long do we need to collect data for a particular building in order to train suitable predictive models. The experiments were performed for all the model structures and for all the different availabilities of future weather data. The performance measures for the models trained by various amounts of training data are shown in Fig. 6 . The lengths of training data are labelled as: $1 \mathrm{~W}, 2 \mathrm{~W}$, $3 \mathrm{~W}, 4 \mathrm{~W}, 5 \mathrm{~W}, 6 \mathrm{~W}, 2 \mathrm{M}, 3 \mathrm{M}, 4 \mathrm{M}, 5 \mathrm{M}, 6 \mathrm{M}$, where $\mathrm{W}$ denotes weeks, and M months. Clearly the linear models require shorter training periods (approximately 6 weeks), whereas, due to their higher complexity, the nonlinear models require about 3 months of training data to reach a good performance. This seems to suggest that, when fitting a predictive model to a new building, linear models may be the first choice in order to obtain useful results already within a month, and later, after several months, more elaborate nonlinear models can be reliably constructed.
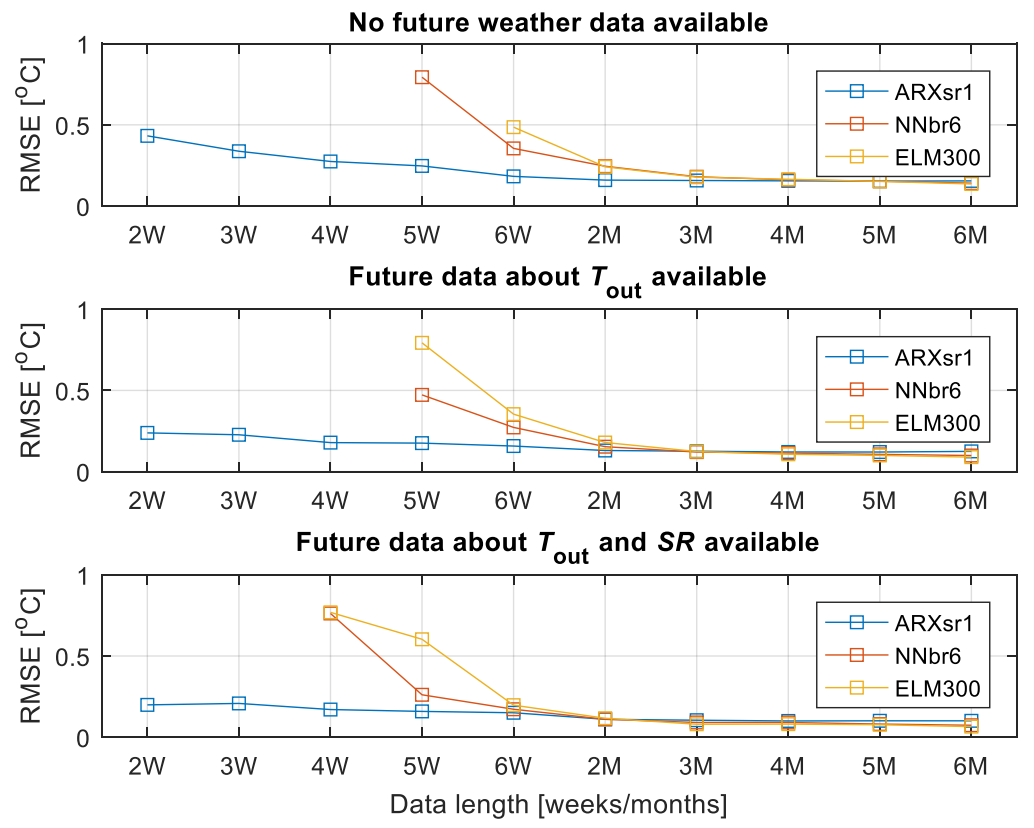

Figure 6: RMSE performance measures for the ARXsr1, NNbr6 and ELM300 models, trained by data of various lengths 


\subsection{Model structures}

Based on the insight obtained from the above-described results regarding the choice of suitable parameters for constructing predictive models, this subsection presents and compares the results for different model structures. The results were obtained by using the embedding dimension $D_{\mathrm{e}}=24 \mathrm{~h}$ for all the models, as well as a data length of $6 \mathrm{M}$ (six months). They are summarized for all the model structures (ARXsr1, ARXsr2, NNlm, NNbr, ELM) and for all the possible future weather conditions in Table 3, and presented in Fig. 7.

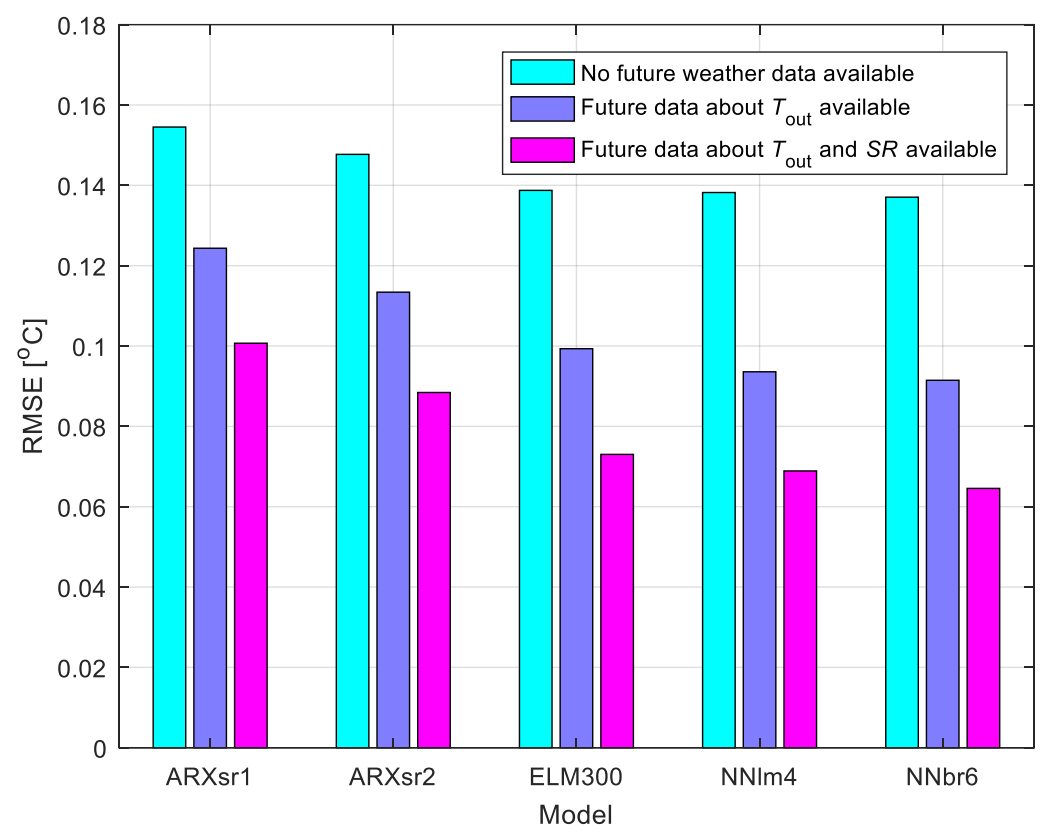

Figure 7: RMSE performance measures for different model structures, and three different sets of future weather conditions

Table 3: RMSE performance measures for different model structures, and three different sets of possible future weather conditions

\begin{tabular}{lccc}
\hline Model & $\begin{array}{c}\text { RMSE }\left[{ }^{\circ} \mathrm{C}\right] \\
\text { Neather data }\end{array}$ & $\begin{array}{c}\text { Future } T_{\text {out }} \\
\text { available }\end{array}$ & $\begin{array}{c}\text { Future } T_{\text {out }} \text { and } \\
S R \text { available }\end{array}$ \\
\hline ARXsr1 & 0.155 & 0.124 & 0.101 \\
ARXsr2 & 0.148 & 0.113 & 0.088 \\
ELM300 & 0.139 & 0.099 & 0.073 \\
NNIm4 & 0.138 & 0.094 & 0.069 \\
NNbr6 & 0.137 & 0.091 & 0.065 \\
\hline
\end{tabular}

A number of observations can be made from these results regarding the model structures:

- Available future weather data considerably improved the predictive performance of all the tested models. Besides the outdoor temperature $\left(T_{\text {out }}\right)$, the availability of data about solar radiation $(S R)$ significantly improves predictions of the future temperature in buildings $\left(T_{\mathrm{in}}\right)$.

- The ARXsr2 model with quadratic terms shows slightly better performance than that of the linear ARXsr1 model, but it is more complex and requires significantly longer computation time.

- Nonlinear models consistently outperform linear models in both fitting and generalization performance, and are therefore the best choice for predictive models. Whereas the ELM model is an easy-to-use, fast and reliable tool, properly designed neural network models can further improve the predictive performance. 
- The choice of the NN structure is not critical, so that already a few hidden layer neurons $\left(N_{\mathrm{h}}=4\right.$ in the case of the LM algorithm, and $N_{\mathrm{h}}=6$ in the case of the BR algorithm) define excellent NN models which consistently generate good predictions. Adding regularization to learning (i.e. in the case of the NNbr6 model) is highly recommended, and results in overall the best predictive performance presented in this study.

\subsection{Prediction examples}

The results section is concluded by some prediction examples which practically illustrate the performance of the constructed models. Figures 8 and 9 show predictions of indoor temperature $T_{\text {in }}$ for the linear ARXsr 1 model and for the neural NNbr6 model, at various prediction horizons $h=\{1,6,12\}$ hours, and for a selected four weeks interval chosen from the 5th data set representing heating optimized with respect to outdoor temperature $T_{\text {out. }}$. The results are based on weather conditions taking into account the availability of future $T_{\text {out }}$ and $S R$ values. The upper plot in both figures shows the weather variables $\left(T_{\text {out }}, S R\right)$ and the control variable ( $T_{\text {heat }}$ ) for this period, and the lower three plots present the measured and predicted values of $T_{\text {in }}$ and the corresponding errors $(e)$ for each prediction horizon. $T_{\text {in }}$ measured represents the average indoor air temperature, as calculated by the TRNSYS emulation. Compared to the linear ARXsr1 model, the neural network model NNbr6 visually generates significantly better predictions, and very good prediction accuracy can be seen especially at the lower prediction horizons, which are also the most relevant for the application in model predictive control schemes.
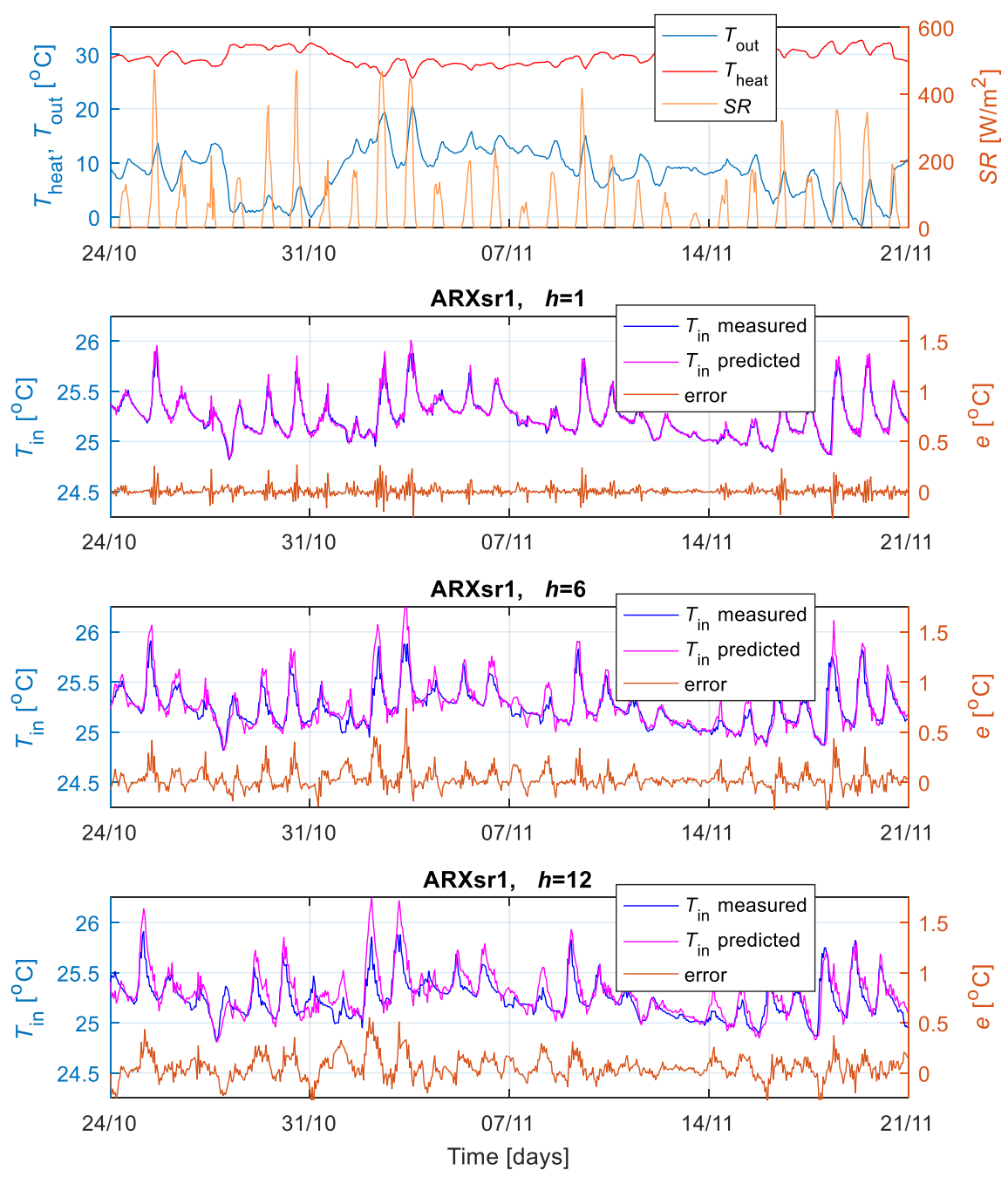

Figure 8: Prediction examples of indoor temperature $T_{\text {in }}$ for a linear model ARXsr1, for prediction horizons $h=\{1,6$,

$12\}$ hours 

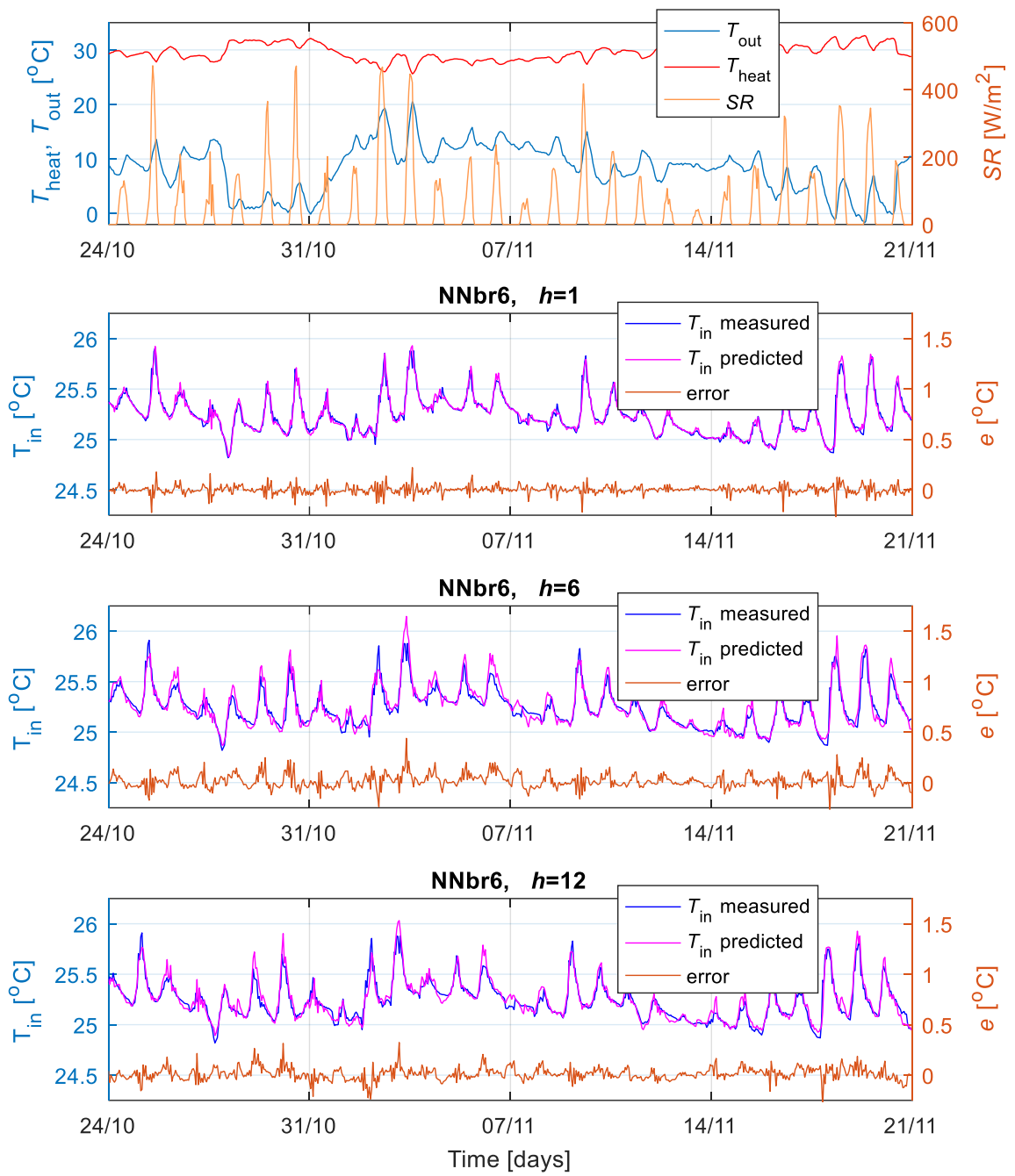

Figure 9: Prediction examples of indoor temperature $T_{\text {in }}$ for a regularized neural network model NNbr6, for the prediction horizons $h=\{1,6,12\}$ hours

\section{Conclusions}

In this paper various possibilities for the development of machine learning based data-driven models for the short-term prediction of indoor temperature in buildings have been investigated. Short-term predictive horizons within the range from 1 hour to 12 hours were considered with the aim of constructing models which can be applied in model predictive control applications. The study was based on a TRNSYS emulation of a residential building heated by a heat pump, combined with measured weather data for a typical winter season in Ljubljana, Slovenia. The study investigated various aspects of the construction of predictive models, i.e. a comparison of various data-driven machine learning models, the effect and relevance of the availability of future weather data, embedding dimensions for past inputs, and the required data lengths for the construction of the models. The conclusions addressing our research objectives can be summarized as follows:

- Model structures: the nonlinear models consistently outperformed the linear models in both their fitting and generalization performance, and are therefore recommended as good predictive models. The best results were obtained by using properly designed neural network models, with only a few hidden layer neurons. Adding regularization to neural learning is highly recommended, and results in overall the best predictive performance in this study (the NNbr6 model).

- Availability of weather data: the availability of future weather data considerably improved the predictive performance of all the tested models. Besides data about the future outdoor temperature $\left(T_{\text {out }}\right)$, data about future expected solar radiation $(S R)$ can significantly improve predictions of temperature in buildings. 
- Embedding dimensions: compared to the nonlinear models, the linear models required longer embedding dimensions for accurate predictions. The linear models converged to stable predictions at $D_{\mathrm{e}}=24$ hours, whereas the nonlinear NN models were not very sensitive to the use of past data although they still benefited by the inclusion of recent data obtained within the last day.

- Training data length: the linear models converged to accurate predictions in shorter training periods (of approximately 6 weeks), whereas, due to their higher complexity, the nonlinear models require about 3 months of training data in order to reach a suitable performance. Thus linear models can be used for the rapid generation of predictive models, and a few months later more advanced nonlinear models can be reliably constructed.

- Prediction errors: The results summarized in Table 3 show that the RMSE prediction errors, averaged across all the data sets and all the prediction horizons, are within the range between $0.155^{\circ} \mathrm{C}$ for the linear ARXsr1 model (in the case of no availability of future weather data), and $0.065^{\circ} \mathrm{C}$ for the neural NNbr6 model (in the case of available future data about $T_{\text {out }}$ and $S R$ ). The neural model trained in the case of available future data about $T_{\text {out }}$ (but without future data about $S R$ ) performed with an RMSE error of $0.091^{\circ} \mathrm{C}$. In a realistic case with less than accurate future outdoor temperature forecasts, this accuracy can be estimated around $\approx 0.1^{\circ} \mathrm{C}$ which can be considered as high accuracy and is therefore appropriate for including such a model in a model predictive control scheme.

The results of this study systematically address and bring new insights into the construction of machine learning based predictive models for the predictive control of heating systems in buildings. They describe the important construction parameters which can be directly implemented in the development of predictive models for different types of buildings.

In future research, more building types and different heating systems will be considered and combined with the operational data of real buildings. An adaptive modelling approach will also be explored, which is mostly suited to more "dynamic" buildings, which may include building renovation, a variable number or residents, changing of the behavior of occupants, etc. Combined heating/cooling systems will also be considered in future research. The limitations of the suggested methodology are mostly based on the inaccuracy of available weather forecasts which influences the predictive accuracy (as summarized in Figure 7). The results of this research are relevant for further improvement of thermal comfort in modern intelligent buildings operated by model predictive controlled heating systems.

\section{Acknowledgements}

This work was supported by ARRS - the Slovenian Research Agency, Research program P2-0241 "Synergetics of complex systems and processes", and was co-financed by the Republic of Slovenia and the European Union under the European Regional Development Fund.

\section{References}

Afroza Z., Shafiullaha GM., Urmeea T., \& Higginsc G. (Article in press). Modeling techniques used in building HVAC control systems: A review. Renewable and Sustainable Energy Reviews, Article in press.

Ahmad T., Chen H., Guo Y., \& Wang J. (2018). A comprehensive overview on the data driven and large scale based approaches for forecasting of building energy demand: A review. Energy and Buildings, 165: 301-320.

Al-Saadi S. N., \& Zhai Z. (2015). A new validated TRNSYS module for simulating latent heat storage walls. Energy and Buildings, 109: 274-290. 
Amasyali K., \& El-Gohary N.M. (2018). A review of data-driven building energy consumption prediction studies, Renewable and Sustainable Energy Reviews, 81: 1192-1205.

Arabzadeh V., Alimohammadisagvand B., Jokisalo J., \& Siren K. (2018). A novel cost-optimizing demand response control for a heat pump heated residential building. Building Simulation, 11: 533-547.

ARSO. (2017). Weather reference year for Ljubljana, Slovenia. Retrieved April 24, 2018, from http://www.meteo.si/met/en/climate/tables/test_ref_year

Bamdad K., Cholette M. E., Guan L., \& Bell J. (2018). Building energy optimisation under uncertainty using ACOMV algorithm. Energy and Buildings, 167: 322-333.

Biswas M. A. R., Robinson M. D., \& Fumo N. (2016). Prediction of residential building energy consumption: A neural network approach. Energy, 117: 84-92.

Cortes C., \& Vapnik V. (1995). Support-Vector Networks. Machine Learning, 20: 273-297.

Crawley D. B., Hand J. W., Kummert M., \& Griffith B. T. (2008). Contrasting the capabilities of building energy performance simulation programs. Building and Environment, 43: 661-673.

Deb C., Zhang F., Yang J., Eang Lee S., \& Wei Shaha K. (2017). A review on time series forecasting techniques for building energy consumption. Renewable and Sustainable Energy Reviews, 74: 902-924.

Ding S., Xu X., \& Nie R. (2014). Extreme learning machine and its applications. Neural Computing and Applications, $25: 549-556$.

Ding Y., Zhang Q., Yuan T., \& Yang K. (2018). Model input selection for building heating load prediction: A case study for an office building in Tianjin. Energy and Buildings, 159: 254-270.

Do H., \& Cetin K.S. (2018). Residential Building Energy Consumption: a Review of Energy Data Availability, Characteristics, and Energy Performance Prediction Methods. Current Sustainable/Renewable Energy Reports, 5: 76-85.

Draper N. R., \& Smith H. (1998). Applied Regression Analysis (3rd ed.). New York: John Wiley \& Sons.

Drissi Lamrhari EH., \& Benhamou B. (2018). Thermal behavior and energy saving analysis of a flat with different energy efficiency measures in six climates, Building Simulation, 11: 1123-1144.

Fan C., Xiao F., \& Zhao Y. (2017). A short-term building cooling load prediction method using deep learning algorithms. Applied Energy, 195: 222-233.

Fan C., Wang J., Gang W., \& Li S. (2019). Assessment of deep recurrent neural network-based strategies for short-term building energy predictions. Applied Energy, 236: 700-710.

Fanger P. O. (1970). Thermal Comfort: Analysis and Applications in Environmental Engineering. New York: McGraw-Hill Inc.

Ferracuti F., Fonti A., Ciabattoni L., Pizzuti S., Arteconi A., Helsen L., \& Comodi G. (2017). Data-driven models for short-term thermal behaviour prediction in real buildings. Applied Energy, 204: 1375-1387.

Foresee F. D., \& Hagan M. T. (1997). Gauss-Newton Approximation to Bayesian Learning. In International Joint Conference on Neural Networks (pp. 1930-1935). Houston, Texas.

Foucquier A., Robert S., Suard F., Stéphan L., \& Jay A. (2013). State of the art in building modelling and energy performances prediction: A review. Renewable and Sustainable Energy Reviews, 23: 272-288.

Gang W., Wang J., \& Wang S. (2014). Performance analysis of hybrid ground source heat pump systems based on ANN predictive control. Applied Energy, 136: 1138-1144.

Guo Y., Wang J., Chen H., Li G., Liu J., \& Xu C. (2018). Machine learning-based thermal response time ahead energy demand prediction for building heating systems. Applied Energy, 221: 16-27.

Hagan M. T., \& Menhaj M. B. (1994). Training Feedforward Networks with the Marquardt Algorithm. IEEE Transactions on Neural Networks, 5: 989-993.

Haykin S. (2009). Neural Networks and Learning Machines (3rd ed.). New Jersey, USA: Pearson.

Hong T., Langevin J., \& Sun K. (2018). Building simulation: Ten challenges. Building Simulation, 11:871-898. 
Huang G.-B., Zhu Q., Siew C., Ã G. H., Zhu Q., Siew C., ... Siew C. (2006). Extreme learning machine: Theory and applications. Neurocomputing, 70: 489-501.

Huang G., Huang G. Bin, Song S., \& You K. (2015). Trends in extreme learning machines: A review. Neural Networks, 61 : $32-48$.

Huang S., Zuo W., \& Sohn M.D. (2018). A Bayesian Network model for predicting cooling load of commercial buildings. Building Simulation, 11: 87-101.

ISO 13790: 2008 Energy performance of buildings - Calculation of energy use for space heating and cooling. (2008). International Organization for Standardization, Technical Committee : ISO/TC 163/SC 2 Calculation methods.

Kalogirou S. A. (2001). Artificial neural networks in renewable energy systems applications: a review. Renewable and Sustainable Energy Reviews, 5: 373-401.

Killian M., \& Kozek M. (2018). Implementation of cooperative Fuzzy model predictive control for an energy-efficient office building. Energy and Buildings, 158: 1404-1416.

Klein S., Beckman W., Mitchell J., Duffie J., Duffie N., \& Freeman T. (2013). TRNSYS 17: a transient system simulation program.

Li Q., Meng Q., Cai J., Yoshino H., \& Mochida A. (2009). Predicting hourly cooling load in the building: A comparison of support vector machine and different artificial neural networks. Energy Conversion and Management, 50: 90-96.

Li X., \& Wen J. (2014). Review of building energy modeling for control and operation. Renewable and Sustainable Energy Reviews, 37: 517-537.

Lindelöf D., Afshari H., Alisafaee M., Biswas J., Caban M., Mocellin X., \& Viaene J. (2015). Field tests of an adaptive, modelpredictive heating controller for residential buildings. Energy and Buildings, 99: 292-302.

Liu X., Gao C., \& Li P. (2012). A comparative analysis of support vector machines and extreme learning machines. Neural Networks, 33: 58-66.

Liu Z., Wu D., Liu Y., Han Z., Lun L., Gao J., Jin G., \& Cao G. (2019). Accuracy analyses and model comparison of machine learning adopted in building energy consumption prediction. Energy Exploration \& Exploitation, Article in press.

Lu S., Zhao Y., Fang K., Li Y., \& Sun P. (2017). Establishment and experimental verification of TRNSYS model for PCM floor coupled with solar water heating system. Energy and Buildings, 140: 245-260.

Magalhães S. M. C., Leal V. M. S., \& Horta I. M. (2017). Modelling the relationship between heating energy use and indoor temperatures in residential buildings through Artificial Neural Networks considering occupant behavior. Energy and Buildings, 151: $332-343$.

Niu F., O’Neill Z., \& O’Neill C. (2018). Data-driven based estimation of HVAC energy consumption using an improved Fourier series decomposition in buildings. Building Simulation, 11:633-645.

Oldewurtel F., Parisio A., Jones C. N., Gyalistras D., Gwerder M., Stauch V., ... Morari M. (2012). Use of model predictive control and weather forecasts for energy efficient building climate control. Energy and Buildings, 45: 15-27.

Pang X., Duarte C., Haves P., \& Chuang F. (2018). Testing and Demonstration of Model Predictive Control Applied to a Radiant Slab Cooling System in a Building Test Facility Demonstration of Model Predictive Control Applied to a Radiant Slab Cooling System in a Building Test Facility. Energy \& Buildings, 172: 432-441.

Pedersen L. (2007). Use of different methodologies for thermal load and energy estimations in buildings including meteorological and sociological input parameters. Renewable and Sustainable Energy Reviews, 11: 998-1007.

Potočnik P., Vidrih B., Kitanovski A., \& Govekar E. (2018). Analysis and optimization of thermal comfort in residential buildings by means of a weather-controlled air-to-water heat pump. Building and Environment, 140: 68-79.

Prívara S., Cigler J., Ván̆a Z., Oldewurtel F., Sagerschnig C., \& Žáčeková E. (2013). Building modeling as a crucial part for building predictive control. Energy and Buildings, 56: 8-22.

Reynders G., Diriken J., \& Saelens D. (2014). Quality of grey-box models and identified parameters as function of the accuracy of input and observation signals. Energy and Buildings, 82: 263-274.

Ríos-Moreno G. J., Trejo-Perea M., Castañeda-Miranda R., Hernández-Guzmán V. M., \& Herrera-Ruiz G. (2007). Modelling temperature in intelligent buildings by means of autoregressive models. Automation in Construction, 16: 713-722. 
Robinson, C., Dilkina B., Hubbs J., Zhang W., Guhathakurta S., Brown M.A., \& Pendyalad R.M. (2017), Machine learning approaches for estimating commercial building energy consumption. Applied Energy, 208: 889-904.

Romero Rodríguez L., Sánchez Ramos J., Álvarez Domínguez S., \& Eicker U. (2018). Contributions of heat pumps to demand response: A case study of a plus-energy dwelling. Applied Energy, 214: 191-204.

Safa A. A., Fung A. S., \& Kumar R. (2015). Heating and cooling performance characterisation of ground source heat pump system by testing and TRNSYS simulation. Renewable Energy, 83: 565-575.

Schmelas M., Feldmann T., \& Bollin E. (2017). Savings through the use of adaptive predictive control of thermo-active building systems (TABS): A case study. Applied Energy, 199: 294-309.

Serale G., Fiorentini M., Capozzoli A., Bernardini D., \& Bemporad A. (2018). Model Predictive Control (MPC) for Enhancing Building and HVAC System Energy Efficiency: Problem Formulation, Applications and Opportunities. Energies, $11: 631$.

Smarra F., Jain A., Rubeis T. De, Ambrosini D., Innocenzo A. D., \& Mangharam R. (2018). Data-driven model predictive control using random forests for building energy optimization and climate control. Applied Energy, 226: 1252-1272.

Soldo B., Potočnik P., Šimunović G., Šarić T., Govekar E. (2019). Improving the residential natural gas consumption forecastingmodels by using solar radiation. Energy and Buildings, 69: 498-506.

Villa-Arrieta M., \& Sumper A. (2018). A model for an economic evaluation of energy systems using TRNSYS. Applied Energy, 215: 765-777.

Wang L., Kubichek R., \& Zhou X. (2018). Adaptive learning based data-driven models for predicting hourly building energy use. Energy and Buildings, 159: 454-461.

Wei Y., Zhang X., Shi Y., Xia L., Pan S., Wu J., Han M., \& Zhao Z. (2018). A review of data-driven approaches for prediction and classification of building energy consumption. Renewable and Sustainable Energy Reviews, 82: 1027-1047.

Yun K., Luck R., Mago P. J., \& Cho H. (2012). Building hourly thermal load prediction using an indexed ARX model. Energy and Buildings, 54: 225-233.

Žáčeková E., Vána Z., \& Cigler J. (2014). Towards the real-life implementation of MPC for an office building: Identification issues. Applied Energy, 135: 53-62.

Zygierewicz A. (2016). Implementation of the Energy Efficiency Directive (2012/27/EU): Energy Efficiency Obligation Schemes. 